\title{
Distinct subtypes of genomic PTEN deletion size influence the landscape of aneuploidy and outcome in prostate cancer
}

\author{
Thiago Vidotto ${ }^{1}$, Daniel Guimarães Tiezzi ${ }^{2}$ and Jeremy A. Squire ${ }^{1,3,4^{*}}$ (D)
}

\begin{abstract}
Background: Inactivation of the PTEN tumor suppressor gene by deletion occurs in 20-30\% of prostate cancer tumors and loss strongly correlates with a worse outcome. PTEN loss of function not only leads to activation of the PI3K AKT pathway, but is also thought to affect genome stability and increase levels of tumor aneuploidy. We performed an in silico integrative genomic and transcriptomic analysis of 491 TCGA prostate cancer tumors. These data were used to map the genomic sizes of PTEN gene deletions and to characterize levels of instability and patterns of aneuploidy acquisition.
\end{abstract}

Results: PTEN homozygous deletions had a significant increase in aneuploidy compared to PTEN tumors without an apparent deletion, and hemizygous deletions showed an intermediate aneuploidy profile. A supervised clustering of somatic copy number alterations (SCNA) demonstrated that the size of PTEN deletions was not random, but comprised five distinct subtypes: (1) "Small Interstitial" (70 bp-789Kb); (2) "Large Interstitial" (1-7 MB); (3) "Large Proximal" (3-65 MB); (4) "Large Terminal" (8-64 MB), and (5) "Extensive" (71-132 MB). Many of the deleted fragments in each subtype were flanked by low copy repetitive (LCR) sequences. SCNAs such as gain at 3q21.1-3q29 and deletions at 8p, RB1, TP53 and TMPRSS2-ERG were variably present in all subtypes. Other SCNAs appeared to be recurrent in some deletion subtypes, but absent from others. To determine how the aneuploidy influenced global levels of gene expression, we performed a comparative transcriptome analysis. One deletion subtype (Large Interstitial) was characterized by gene expression changes associated with angiogenesis and cell adhesion, structure, and metabolism. Logistic regression demonstrated that this deletion subtype was associated with a high Gleason score ( $H R=2.386 ; 95 \%$ C.I. 1.245-4.572), extraprostatic extension ( $H R=2.423,95 \%$ C.I. 1.157-5.075), and metastasis ( $H R=7.135 ; 95 \%$ C.I. 1.540-33.044). Univariate and multivariate Cox Regression showed that presence of this deletion subtype was also strongly predictive of disease recurrence.

Conclusions: Our findings indicate that genomic deletions of PTEN fall into five different size distributions, with breakpoints that often occur close LCR regions, and that each subtype is associated with a characteristic aneuploidy signature. The Large Interstitial deletion had a distinct gene expression signature that was related to cancer progression and was also predictive of a worse prognosis.

Keywords: TCGA, Prostate cancer, Genomic instability, PTEN, Chromosome 10

\footnotetext{
* Correspondence: jsquireinsp@gmail.com

${ }^{1}$ Department of Genetics, Ribeirão Preto Medical School, University of São Paulo, Ribeirão Preto, Brazil

${ }^{3}$ Department of Pathology and Legal Medicine, Ribeirão Preto Medical

School, University of São Paulo, 3900 Bandeirantes Avenue, Monte Alegre,

Ribeirão Preto, São Paulo 14040-900, Brazil

Full list of author information is available at the end of the article
} 


\section{Background}

Prostate Cancer is the most frequent solid tumor in men and is the third most common cancer type in the world [1]. Genomic deletion of the PTEN tumor suppressor gene occurs in $20-30 \%$ of prostate cancer tumors, and presence of this aberration strongly correlates with a worse outcome [2-5]. There is therefore increasing interest in the use of loss of the PTEN gene and its protein as a predictive biomarker of outcome [5-7]. Moreover, PTEN loss is associated with increased levels of chromosomal instability [8] and the accumulation of high levels of aneuploidy in tumors [9].

The occurrence of aneuploidy, arising as a consequence of genomic instability, is one of the most prominent features of human cancers [10]. Through clonal expansion, tumors often acquire high levels of sequence mutations together with numerical and structural chromosomal rearrangements due to loss of integrity in the DNA repair machinery. In this way, these defects in the genome and chromosome maintenance may also provide a selectively advantageous progression for the malignant cells [11].

The PTEN gene is located at $10 \mathrm{q} 23.31$ and mapping studies have shown that PTEN genomic deletions in prostate cancer vary in size from a few hundred kb of DNA to several Mb. Interestingly, PTEN deletions often appear to have breakpoints that initiate close to low copy repeat (LCR) regions [12]. The LCR repetitive elements (also known as segmental duplications) are unstable DNA sequences that are represented two or more times in the genome with high sequence identity, but not arising by retrotransposition [13]. On chromosome 10 there is one LCR hotspots $400 \mathrm{~kb}$ centromeric of PTEN that may facilitate the inter- and intragenomic alterations leading to PTEN loss $[14,15]$. LCRs can promote the occurrence of somatic copy number alterations (SCNAs) through non-allelic homologous recombination (NAHR), non-homologous end-joining (NHEJ), and fork stalling and template switching (FoSTeS) [16-19]. To date, PTEN gene deletions have been extensively analyzed through FISH assays [4, 5, 20, 21], but a detailed mapping of chromosome 10 deletions that span PTEN and their impact on SCNAs and levels of aneuploidy in prostate cancer outcome have not been investigated in detail [22, 23].

This study was designed to determine whether the observed variations in the size of PTEN genomic deletions has an impact on overall levels of genomic instability and the acquisition of aneuploidy in the prostate cancer genome. Our study design also addresses whether the initiation of deletion events is influenced by the proximity of LCR elements along chromosome 10 and whether deletion size correlates with any clinical features associated with prostate cancer progression.

\section{Results}

Impact of homozygous and Hemizygous PTEN deletions on genomic instability and aneuploidy

We identified homozygous or hemizygous PTEN gene deletions in $118 / 491(24.1 \%)$ of the prostate tumors and the regions of genomic loss varied in length from $70 \mathrm{bp}$ to $132 \mathrm{MB}$. Overall we found that $44 / 491$ (9\%) had homozygous PTEN deletions and 74/491 (15.1\%) had hemizygous deletions. Since about $5 \%$ of prostate cancers inactivate a PTEN allele by a somatic point mutation (frameshift deletions and insertions, in-frame deletions, missense mutations, or splice-site mutation) [24] and not by a large genomic deletion, it was necessary to consider the effect of any mutation caused by sequence alterations. We found that $66 \%$ of tumors with hemizygous genomic deletions also harbored somatic mutations in the remaining PTEN allele. Such tumors would be expected to express no PTEN protein. In contrast, when there is a hemizygous deletion but the remaining PTEN gene appears to be undeleted (PTEN intact), the protein expression levels may be reduced so that functional haploinsufficiency may occur (discussed below).

To evaluate the impact of homozygous vs. hemizygous PTEN deletions on genomic instability and aneuploidy, we performed a Kruskal-Wallis test considering the total number of SCNAs, the percentage of genome altered, the total number of mutations, and the MATH tumor heterogeneity score. Tumors with PTEN homozygous deletions had a higher number of SCNA $(P$-value $<0.0001)$, increased aneuploidy (percentage of genome altered, $P$-value $<0.0001)$, and an increased number of mutations $(P$-value $=0.015)$. The loss of one copy of the PTEN gene was sufficient to affect levels of instability since hemizygous deletions demonstrated significant differences when compared to PTEN intact (Additional file 1).

\section{The different sizes of PTEN genomic deletions influence the SCNA landscape and pattern of aneuploidy in prostate cancer}

To determine whether the deletions had non-random size distributions along chromosome 10, we performed a supervised clustering of all the SCNA leading to PTEN deletion. This analysis demonstrated that there were five distinct deletion subtypes classified as: (1) Small Interstitial (size range $70 \mathrm{bp}-789 \mathrm{~Kb}$ ); (2) Large Interstitial (1-7 MB); (3) Large Proximal (3-65 MB); (4) Large Terminal (8-64 MB), and (5) Extensive (71-132 MB) (Fig. 1). The deletion subtypes presented similar proportions of hemi- and homozygous deletions (Additional file 2). The list of all genes present in the regions of chromosome 10 loss for each deletion subtype is shown in Additional file 3.

Many of the deletions breakpoints occurred close to genomic regions containing LCRs (see Fig. 1). 


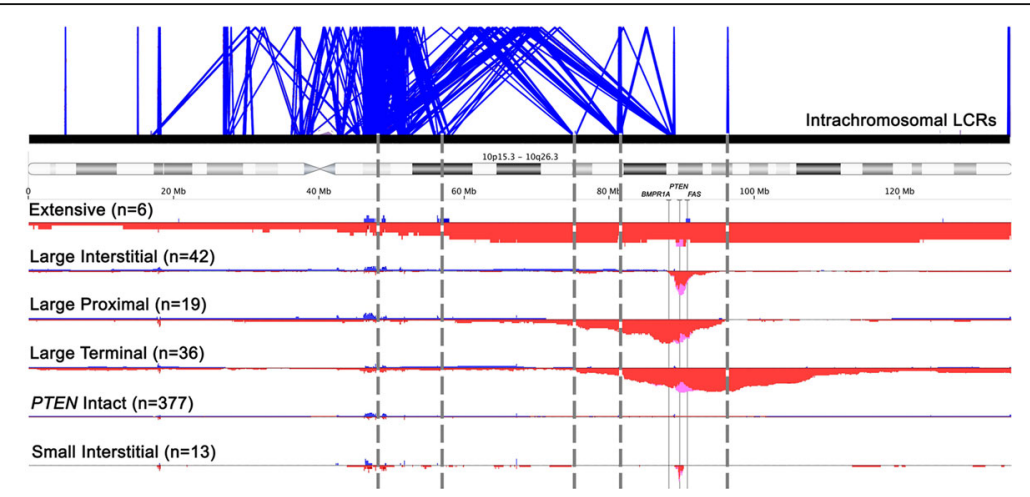

Fig. 1 Chromosome 10 characterization and LCR mapping of the different PTEN deletion subtypes in prostate cancer. The panel below the schematic map of chromosome 10 demonstrates the different deletion subtypes and their frequency for each group. The genome is displayed horizontally, and the frequency of somatic copy number alterations (SCNA) at any given location are displayed on the $y$-axis. Red, pink and blue indicate the frequencies as a percentage of hemizygous deletions, homozygous deletions, and gains, respectively. The three thin continuous vertical lines show the precise location of the genes BMPRIA, PTEN, and FAS genes. The number of deletions for each subtype is shown in parentheses. The tumors that have PTEN intact are also shown. The panel above shows the intrachromosomal LCR regions along chromosome 10 with related regions of homology linked by thin blue lines. The five grey vertical dashed lines identify clusters of LCRs that map to the vicinity of PTEN deletion breakpoints defined by copy number transitions. Many deletions appear to originate at the small LCR cluster in between PTEN and BMPR1A. Mapping was performed using data from the Segmental Duplication database (http://humanparalogy.gs.washington.edu) for sequences with more or equal to $5 \mathrm{~Kb}$ and showing equal or more similarity in $90 \%$ of the duplicated sequence

Additionally, the breakpoint regions of all deletion subtypes showed a high number of flanking LCRs having $>1 \mathrm{~Kb}$ and $90-99 \%$ similarity levels in both upper and lower extremities of the deleted fragments (manuscript in preparation).

To determine if the five PTEN deletion subtypes had distinct patterns of aneuploidy, we compared their SCNA landscapes to overall levels of copy number change in tumors without an apparent PTEN gene loss (Fig. 2). Some of the imbalances such as gain at 3q21.1$3 \mathrm{q} 29$ and deletions at $8 \mathrm{p}, R B 1, T P 53$, and TMPRSS2 were found with varying incidences in all five subtypes. The 3q21.1-3q29 region has eight cancer-related genes: PIK3CA, ZNF9, FOXL2, ATR, WWTR1, GMPS, MLF1, and TBLIXR1. Other SCNAs appeared to be enriched in some subtypes and not in others. For example, both the Small and Large Interstitial deletion subtypes were characterized by having gains of chromosome 7 . The Large Terminal, Proximal and Extensive had losses of chromosome 6. The Small Interstitial deletion was the only subtype to have extensive gains of chromosome 11. The Extensive deletions had the largest region of copy number loss and were characterized by concurrent deletions of chromosome 12p, 18q, whole chr13, and gains at 5p11 (Fig. 2).

\section{The effect of the different PTEN deletion subtypes on genomic instability and the somatic mutation rate in prostate cancer}

When comparing the five PTEN deletion subtypes to the tumors without apparent PTEN loss, the Large Terminal and Large Interstitial deletion subtypes exhibited a significant increase in the total number of SCNAs. Moreover, we observed that Large Proximal and Large Interstitial demonstrated increased levels of mutations and that all deletion subtypes except Small Interstitial exhibited a significant increase in the percentage of genome altered (Fig. 3).

We then investigated whether tumors with concomitant PTEN hemizygous deletion and a somatic mutation in the remaining allele would lead to a more significant impact in aneuploidy. We observed that patients with both hemizygous deletions and somatic mutations demonstrated high levels of aneuploidy (percentage of genome altered, $P$-value $=0.008)$, total number of SCNAs $(P$-value $<0.0001)$, and total number of mutations $(P$-value $=0.05)$ when compared to PTEN intact and tumors with both alleles present with a somatic mutation in one of the alleles (Additional file 4).

MutSigCV analysis presented the 19 most differentially mutated genes across the cases: $C D K N 1 B$, FBXO46, FRG1, GAST, KIAA1257, LCE1F, MLF2, PTEN, SNRNP27, SPOP, TMEM211, YWHAQ, TP53, FOXA1, ZMYM3, KDM6A, RYBP, SMARCA1, and ZFHX3. To determine whether PTEN hemi- and homozygous deletions impact the mutational signatures of the 19 genes, a chi-square was performed. Differences in TP53, SPOP, and PTEN gene mutations $(P$-value $<0.001)$ were observed. TP53 mutations were present in $16 \%$ and $27 \%$ in tumors with hemi- and homozygous deletions of PTEN, respectively. SPOP mutations were present in $3 \%$ of hemi- and $3 \%$ of homozygous deletion tumors and in $94 \%$ of PTEN intact tumors. 


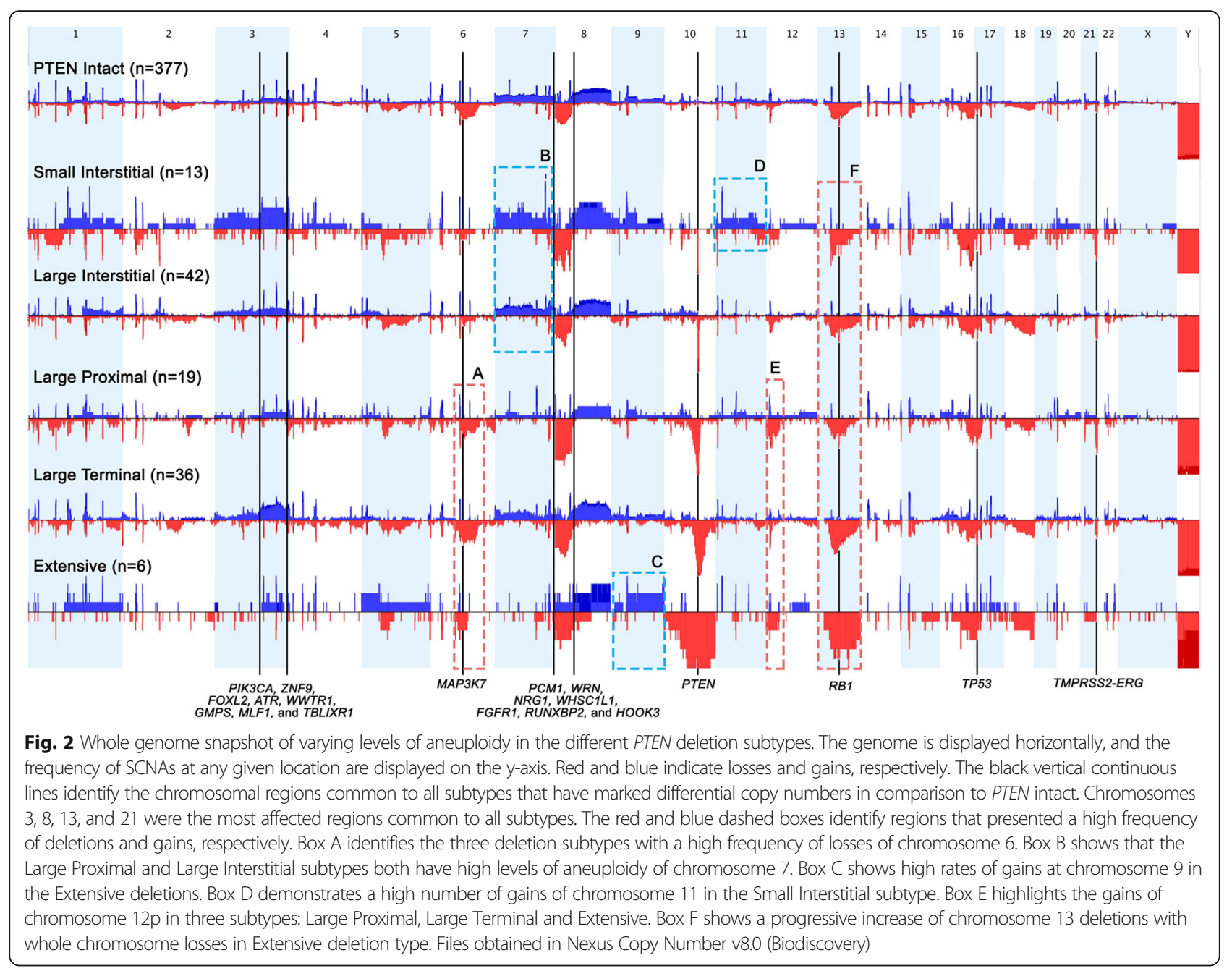

When we compared the frequency of mutation in the 19 genes across the PTEN deletion subtypes to the frequency in the PTEN intact tumors, we identified significant differences for TP53 (P-value $=0.0001), \quad S P O P$ $(P$-value $=0.013)$, and YWHAQ $(P$-value $=0.0001)$ genes. In addition, the Large Interstitial type presented the higher number of mutations in TP53 (20\%) when compared to the other deletion subtypes.

\section{Effects of PTEN deletion subtypes on differential gene expression}

Initially, we checked the RNAseq dataset to confirm that when the PTEN gene was deleted the PTEN transcript level was decreased as expected. These analyses showed that PTEN homozygous deletions presented the lowest PTEN mRNA expression value, followed by PTEN hemizygous deletions $(P$-value $<0.0001)$ (Additional file $5 \mathrm{a}$ ). In comparison to PTEN intact tumors, the average for PTEN mRNA expression was significantly decreased for all PTEN deletion subtypes $(P$-value $<0.0001)$, but there were no differences in the relative levels of PTEN mRNA expression across the five deletion subtypes (Additional file $5 b$ ).

To determine how the different genomic sizes of the PTEN deletions can affect global levels of gene expression levels, we performed a group transcriptome comparison of all five subtypes to the expression observed in the tumors without a PTEN deletion. The Large Interstitial deletion subtype was the most different, with 1073 differentially expressed genes in comparison to PTEN intact tumors. The Large Proximal and Large Terminal deletions presented with 197 and 248 differentially expressed genes, respectively. Extensive and Small Interstitial losses had less marked differences with 50 and just seven differentially expressed genes.

Enrichment analysis of the differentially expressed genes from all PTEN deletion subtypes showed that only Large Interstitial and Large Proximal deletions significantly demonstrate alterations of cancer-related pathways (Fig. 4). We observed that Large Interstitial deletions influence the gene expression profile of 

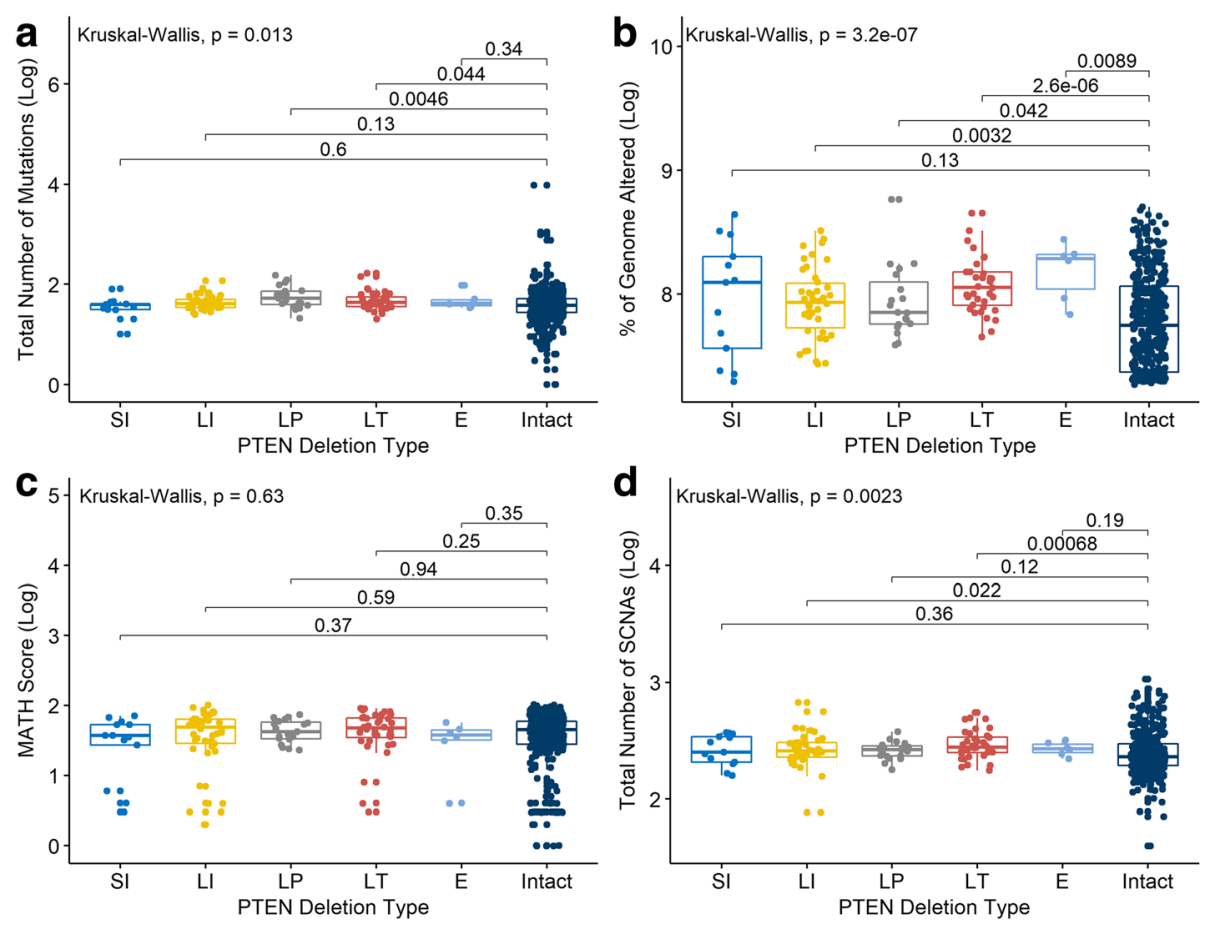

Fig. 3 Variation of mutation, tumor heterogeneity, aneuploidy, and genomic instability in PTEN deletion subtypes in prostate cancer. The boxplots show $\mathbf{a}$ - the total number of mutations, $\mathbf{b}$ - aneuploidy, as percentage of genome altered, $\mathbf{c}$ - Mutant-Allele Tumor Heterogeneity (MATH) score, and $\mathbf{d}$ - total number of SCNAs. The different deletion subtypes show increased heterogeneity for all evaluated parameters. PTEN intact tumors also show increased heterogeneity, with a significant number of outliers. SCNA - somatic copy number alteration, SI - Small Interstitial, LI - Large Interstitial, LP - Large Proximal, LT - Large Terminal, E - Extensive

proteins associated with angiogenesis (e.g., VEGF, SAT1, EMCN, CAV1, HTATIP2, NRP1, CSPG4, PDE3B, ANPEP, and TNFSF12), and cell metabolism (e.g., POLR1B, AMPD3, PGM2, POLD4, PDE2A, NUDT9, NT5M), adhesion (MCAM, JAM3, COMP, NOV, ICAM1, ITGA11, ADAM17, and ADAM9) migration (e.g., PRKD1, LAMC2, SEMA3B, PDGFD, TRIP6, LAMB1, and F2R) and structure (e.g., KCNC2, CTNNAL1, SLC44A1, ADCY1, SLC22A18, EFNA3, UTRN, CSPG4, SLC7A8, KIAA1324, and LPAR3). Moreover, Large Proximal deletions show influence on the expression of genes related to cell metabolism (e.g, OVGP1, UGDH, GAA, GLO1, and GLB1) and structure (e.g., FZD8, ACER3, FAM198B, RAB43, GNPTAB, and CLSTN3) (Fig. 4).

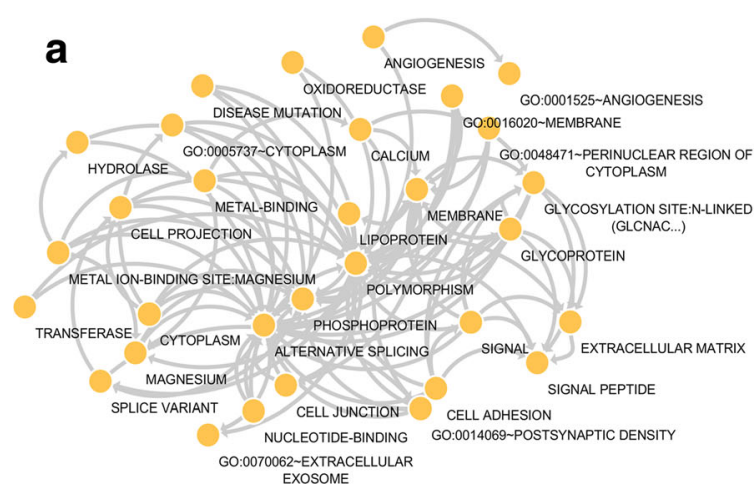

b

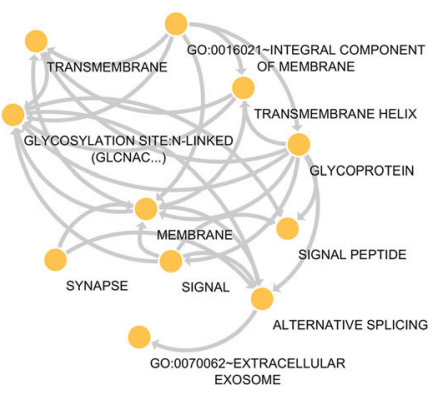

Fig. 4 Enrichment analysis of gene expression in deletion subtypes with extensive aneuploidy. Two PTEN deletion subtypes had distinctive patterns of aneuploidy and were significantly enriched for pathways related to cancer progression. The Large Interstitial subtype (a) was enriched for metabolism, cell structural adhesion and also angiogenesis. The Large Proximal deletions (b) showed effects in pathways associated with cell metabolism and structure. Enrichment analysis was performed through DAVID database and nodes were generated through Enrichment Map (Cytoscape) 
Large interstitial deletions of chromosome 10 that harbor PTEN gene predicts worse outcome in prostate cancer In order to identify the effect of the different PTEN deletion subtypes on clinical and pathological features of prostate cancer, we performed a Chi-square test for race, Gleason score, presence of extraprostatic extension, lymph node invasion, pathologic grade, presence of metastasis, and disease recurrence. PTEN hemi- and homozygous deletions showed significant effects on all investigated clinical parameters (data not shown). For the different deletion subtypes, we observed differences for lymph node invasion ( $P$-value $<0.0001)$, presence of seminal vesicle invasion $(P$-value $=0.003)$, presence of extraprostatic extension $(P$-value $=0.002)$, race $(P$-value $<0.0001)$, and a trend for pathological M $(P$-value $=0.08)$ (Additional file 6). Among the deletion subtypes, Large Interstitial deletions showed the higher rates of extraprostatic extension (36\%), seminal vesicle invasion (41\%), lymph node invasion (36\%), metastasis (75\%), and disease recurrence (46\%) (Additional file 6). We did not observe any differences in time to disease recurrence $(P$-value $=0.69)$ and age at diagnosis $(P$-value $=0.10)$ for the PTEN deletion subtypes, but there was a trend towards men of African-American ancestry having a lower overall incidence of PTEN deletions ( $P$-value $<0.0001)$.

We then performed a univariate logistic regression analysis to investigate whether deletion subtype could significantly predict the occurrence of tumors with high Gleason score $(\geq 8)$, extraprostatic extension, metastasis, and disease recurrence. We observed that Large Interstitial deletions ( $P$-value $=0.009 ; \quad H R=2.386 ;$ C.I. $95 \%$ $1.245-4.572)$ significantly predict higher Gleason scores (Table 1). In addition, Large Interstitial ( $P$-value $=0.019$; $\mathrm{HR}=2.423$; C.I. 95\% 1.157-5.075) and Large Proximal deletions $\quad(P$-value $=0.014 ; \quad H R=6.436 ; \quad$ C.I. $\quad 95 \%$ 1.466-28.260) predict the occurrence of extraprostatic extension in patient samples. Similarly, Small Interstitial $(P$-value $=0.03 ; \mathrm{HR}=3.431 ;$ C.I. 95\% 1.119-10.412), Large Interstitial $(P$-value $=0.001 ; \mathrm{HR}=2.660$; C.I. 95\% 1.389 5.091), and Large Proximal deletions $(P$-value $=0.04$; $\mathrm{HR}=2.633$; C.I. $95 \% 1.038-6.677)$ predict the occurrence of seminal vesicle invasion. Large Interstitial deletions also predict the occurrence of metastasis $(P$-value $=0.01 ; \quad$ HR $=7.135 ; \quad$ C.I. $95 \%$ 1.540-33.044 $)$ (Table 1).

Kaplan Meyer and log-rank analysis showed a significant difference between tumors with PTEN homozygous deletions, PTEN hemizygous deletions, and PTEN intact for the prediction of earlier disease recurrence events $(P$-value $=0.002)$ (Additional file 7a). In addition, Kaplan Meyer curves and log-rank analysis were performed for disease recurrence and demonstrated no significance in the curve for the different PTEN deletion subtypes $(P$-value $=0.11) \quad($ Additional file $7 \mathrm{~b})$. Univariate Cox
Table 1 Univariate logistic regression results for clinical parameters considering the five PTEN deletion subtypes. PTEN intact was set as a baseline in the model. Large Interstitial deletions are reliable predictors of high Gleason scores, extraprostatic extension, and metastasis. The model represents the occurrence of each event analyzed. High Gleason score was obtained through dichotomization: Gleason scores $\leq 7$ were considered as low, and $\geq 8$ were considered as high. * Significant $P$-value $<0.05$

\begin{tabular}{|c|c|c|c|c|}
\hline \multirow[t]{2}{*}{ Clinical Feature } & \multirow[t]{2}{*}{$P$-value } & \multirow{2}{*}{$\begin{array}{l}\text { Odds } \\
\text { Ratio }\end{array}$} & \multicolumn{2}{|c|}{ 95\% C.I. } \\
\hline & & & Lower & Upper \\
\hline \multicolumn{5}{|l|}{ High Gleason Score } \\
\hline Small Interstitial & 0.26 & 1.893 & 0.624 & 5.744 \\
\hline Large Interstitial & $0.009^{*}$ & 2.386 & 1.245 & 4.572 \\
\hline Large Proximal & 0.91 & 0.946 & 0.364 & 2.460 \\
\hline Large Terminal & 0.45 & 1.298 & 0.651 & 2.587 \\
\hline Extensive & 0.057 & 8.112 & 0.938 & 70.137 \\
\hline PTEN Intact & 0.00 & 0.616 & & . \\
\hline \multicolumn{5}{|c|}{ Extraprostatic Extension } \\
\hline Small Interstitial & 0.16 & 2.524 & 0.683 & 9.322 \\
\hline Large Interstitial & $0.01^{*}$ & 2.423 & 1.157 & 5.075 \\
\hline Large Proximal & $0.01^{*}$ & 6.436 & 1.466 & 28.260 \\
\hline Large Terminal & 0.31 & 1.451 & 0.701 & 3.005 \\
\hline Extensive & 0.99 & & 0.000 & . \\
\hline PTEN Intact & 0.00 & 1.321 & & . \\
\hline \multicolumn{5}{|l|}{ Metastasis } \\
\hline Small Interstitial & . & . & & . \\
\hline Large Interstitial & $0.01^{*}$ & 7.135 & 1.540 & 33.044 \\
\hline Large Proximal & . & . & . & . \\
\hline Large Terminal & 0.38 & 2.650 & 0.288 & 24.364 \\
\hline Extensive & & . & . & . \\
\hline PTEN Intact & 0.00 & 0.011 & & . \\
\hline \multicolumn{5}{|c|}{ Seminal Vesicle Invasion } \\
\hline Small Interstitial & $0.03^{*}$ & 3.431 & 1.119 & 10.412 \\
\hline Large Interstitial & $0.001^{*}$ & 2.660 & 1.389 & 5.091 \\
\hline Large Proximal & $0.04^{*}$ & 2.633 & 1.038 & 6.677 \\
\hline Large Terminal & 0.98 & 1.013 & 0.458 & 2.239 \\
\hline Extensive & 0.19 & 2.926 & 0.580 & 14.744 \\
\hline PTEN Intact & 0.00 & 0.342 & & . \\
\hline
\end{tabular}

Regression analysis showed that Large Interstitial deletions are significantly associated with increased chance of disease recurrence $(P$-value $=0.04 ; \mathrm{HR}=1.845$; C.I. 95\% 1.012-3.367) (Table 2).

We then investigated the influence of genomic instability parameters on the likelihood of disease recurrence through univariate Cox Regression. We only found Significant associations were observed for the percentage of genome altered, showing that increased levels of aneuploidy may predict prostate cancer disease 
Table 2 Univariate and multivariate Cox Regression analysis for disease recurrence considering the five PTEN deletion subtypes. Multivariate analysis exhibits age-adjusted results. PTEN intact was set as the baseline for the model. * Significant $P$-value $<0.05$

\begin{tabular}{|c|c|c|c|c|c|c|c|c|c|}
\hline \multirow[t]{3}{*}{ Univariate } & \multirow{3}{*}{$P$} & \multirow{3}{*}{$\mathrm{HR}$} & & & \multirow[t]{3}{*}{ Multivariate } & \multirow{3}{*}{$P$} & \multirow{3}{*}{$\mathrm{HR}$} & \multirow{2}{*}{\multicolumn{2}{|c|}{ 95\% C.I. }} \\
\hline & & & \multicolumn{2}{|c|}{ 95\% C.I. } & & & & & \\
\hline & & & Lower & Upper & & & & Lower & Upper \\
\hline Small Interstitial & 0.32 & 0.373 & 0.052 & 2.694 & Small Interstitial & 0.38 & 0.419 & 0.058 & 3.039 \\
\hline Large Interstitial & $0.04^{*}$ & 1.845 & 1.012 & 3.367 & Large Interstitial & $0.04^{*}$ & 1.844 & 1.007 & 3.377 \\
\hline Large Proximal & 0.72 & 1.239 & 0.387 & 3.960 & Large Proximal & 0.74 & 1.213 & 0.379 & 3.881 \\
\hline Large Terminal & 0.13 & 1.705 & 0.845 & 3.440 & Large Terminal & 0.19 & 1.587 & 0.785 & 3.208 \\
\hline Extensive & 0.13 & 2.955 & 0.719 & 12.153 & Extensive & 0.22 & 2.448 & 0.584 & 10.258 \\
\hline PTEN Intact & 0.26 & . & . & . & PTEN Intact & 0.38 & & & . \\
\hline Age & 0.15 & 1.024 & 0.991 & 1.057 & Age & 0.18 & 1.022 & 0.990 & 1.055 \\
\hline Percentage of genome altered & $0.009^{*}$ & 1.745 & 1.147 & 2.654 & Percentage of genome altered & $0.02^{*}$ & 1.629 & 1.065 & 2.491 \\
\hline
\end{tabular}

PTEN intact was set as a baseline in the model. Percentage of genome altered was dichotomized in high (>average) and low ( $\leq$ average)

recurrence $(P$-value $=0.009 ; \mathrm{HR}=1.745 ;$ CI 95\% $1.147-$ 2.654). Finally, age-adjusted Cox Regression models showed that the presence of the Large Interstitial deletion subtype and an increased percentage of genome altered together were predictive of disease recurrence (Table 2).

\section{Discussion}

To date, PTEN gene and protein have been widely investigated as biomarkers of prognosis in prostate cancer [5, $12,25,26]$. However, since PTEN deletions may also influence the stability of the genome, it is important to determine how PTEN loss influences SCNAs and affects aneuploidy levels in tumors.

The mechanism of PTEN genomic deletion is poorly understood. Chromosome 10 presents a large number of LCRs that increase the chances that intra- or interchromosomal rearrangements may occur. Moreover, many of these LCRs cluster both proximal and distal to the PTEN gene at 10q23.31, and these unstable regions may facilitate the genomic rearrangements leading to deletion events [12]. In this study, we observed five deletion subtype distributions that are flanked by many LCR hotspots, which may initiate of the chromosomal rearrangements leading to gains, losses and the recombination events of chromosome 10 [27, 28].

In prostate cancer, whole genome mate-pair sequencing has shown that the 10q23.31 region has many complex intrachromosomal and interchromosomal rearrangements [22]. Our comparative SCNA analysis showed that large chromosome 10 deletions (Extensive deletions) are linked to increased aneuploidy levels in prostate cancer. Whole chromosome aberrations may occur through defects on mitosis checkpoints, centromere overduplication, and cohesion defects in sister chromatids that may lead to missegregation during mitosis and resulting in an altered SCNA landscape of tumor samples [29]. In addition, the presence of whole chromosome alterations may trigger secondary chromosomal aberrations during tumor progression due to improper cytokinesis, which leads to frequent DNA double-strand breaks that are incorrectly repaired by non-homologous end joining (NHEJ) repair machinery $[11,16,29]$. Concomitantly, the whole chromosome 10 deletion may also independently initiate the dysregulation of the cell cycle, centromere stability and DNA double-strand repair maintained by PTEN [30, 31].

In the cytoplasm, PTEN acts dephosphorylating PIP3, which leads to decreased cell survival, growth and proliferation through the AKT/mTOR axis. Furthermore, in the nucleus, PTEN can downregulate MAPK (ERK-P), promoting the G0-G1 arrest due to cyclin D1 regulation [32], and also upregulate RAD51 expression, which promotes double-stranded-break repair [30]. The PTEN protein can also interact with CENP-C to enhance centromere stability and overall genomic stability [30]. Conversely, PTEN deletions and protein loss are associated with increased copy number alterations and higher levels of aneuploidy in prostate cancer [9]. Taken together, these data demonstrate that PTEN influences cell proliferation and survival, in addition to having a role in the maintenance of genomic and chromosomal stability.

Genomic instability has a critical role in the creation of variants within tumor cell populations, leading to clonal evolution, inter- and intratumoral heterogeneity and therapeutic resistance [11]. By considering genomic instability parameters, we observed that PTEN homozygous deletions demonstrated a significant increase in the total number of SCNA, increased aneuploidy, and total number of mutations when compared to PTEN intact samples. Additionally, PTEN hemizygous deletions showed an intermediate aneuploidy profile. For the PTEN deletion subtypes, we only found that Large Terminal deletions presented an increased total number 
of SCNA and higher aneuploidy levels when compared to PTEN intact tumors.

It has been proposed that the haploinsufficiency of tumor suppressor genes can increase cell proliferation rates that consequently could promote the accumulation of mutations and increased aneuploidy in the genome [33]. Furthermore, hemizygous deletions that harbor proliferation inhibitory genes are thought to be preferentially selected during tumor development [34]. This would be in keeping with mouse studies, which have shown that hemizygous deletion of the Pten C-terminal domain promotes genomic instability and leads to preferential rearrangements at fragile sites [35]. Thus, when both PTEN alleles are lost, the genome of prostate cancer may be significantly impacted due to the complete absence of cell cycle regulation, double-strand break repair, centromere stability, as well as increased cell proliferation rates mediated by the $\mathrm{AKT} / \mathrm{PI} 3 \mathrm{~K} / \mathrm{mTOR}$ and NF- $\kappa B$ signaling pathways [30, 31, 36, 37].

In this study, the Large Interstitial deletion subtype showed the most significant influence on prostate cancer outcome compared to other deletion subtypes. This deletion type presented a distinct profile in most of the investigated parameters. Large Interstitial deletions influence pathways associated with angiogenesis, cell structure, metabolism, adhesion, and migration. Altered cell adhesion is strongly related to tumorigenesis and tumor differentiation [38], increased invasive and metastatic potential [39] and associated with tumor cell stemness [40]. Moreover, Large Interstitial deletions exhibit altered cell structure, being concordant with the observation that these cells might be less differentiated [10]. Such mechanisms are in agreement with our finding that tumors with Large Interstitial deletions showed increased invasive non-organ confined disease, defined by high rates of extraprostatic extension and seminal vesicle invasion. Additionally, altered angiogenesis may promote an increased tumorigenic potential in these tumors [10], since these changes will affect the tumor microenvironment, which could in turn influence the immune cell infiltration profile and extracellular matrix remodelation [41].

Remarkably, the tumors with Large Interstitial deletions also had high rates of TP53 mutations. Pten/Tp53 null murine models of prostate cancer have reduced ARdependent gene expression and altered cell metabolism [42]. Similarly, for human TP53 mutated prostate tumors, there is a strong association with poor outcome [43]. However, TP53 inactivation alone does not lead to genomic instability in physiological conditions [44]. Perhaps collectively the haploinsufficiency of PTEN, together with the other flanking genes present in Large Interstitial deletions, and with TP53 inactivation, may result in reduced apoptosis rates and senescence escape in a replicative stress condition $[45,46]$.
The haploinsufficiency of the genes located in Large Interstitial deletions are also related to cancer development and progression. KLLN, which shares a promoter region with PTEN, promotes cell cycle arrest and apoptosis. In addition, KLLN gene deletions are linked to high risk for thyroid [47] and breast cancer [48]. FAS gene loss of function is also associated with dysregulated apoptosis in vitro [49]. In this way, we suggest that the haploinsufficiency of the genes present in Large Interstitial deletions may drive TP53 inactivation and consequently an acquisition of a greater level of aneuploidy.

Interestingly, we observed that men of AfricanAmerica ancestry might have a lower overall incidence of PTEN deletions. However, due to the predominantly Caucasian representation in the TCGA cohort, a detailed investigation of deletion size in the context of racial origins could not be conducted. This type of study could be performed on a cohort with more mixed racial origins. It has recently been shown that primary prostate tumors arising in African-Americans have reduced rates of PTEN loss when compared to tumors of EuropeanAmerican patients [50-52]. Moreover, the association between PTEN loss and poor prognosis appears to be independent of racial ancestry [52].

\section{Conclusion}

These findings allow us to hypothesize on both the order of genomic events and the impact on aneuploidy when PTEN becomes deleted in prostate cancer. It is possible that the acquisition of the initial hemizygous PTEN deletions or mutations may increase levels of genomic instability because of protein haploinsufficiency. The presence of clusters of microhomology at LCR regions along chromosome 10 may then facilitate second genomic deletion events that remove the remaining functional PTEN allele in the five characteristic size distributions that we observed. The Large Interstitial deletion subtype appears to have a distinct pattern of aneuploidy and gene expression changes that confer more aggressive disease. Collectively, PTEN genomic deletions may thus not only lead to activation of the PI3K/ AKT pathway, but the size of the deletion events themselves may influence gene expression and the levels of acquired aneuploidy.

\section{Methods}

\section{Cohort and data description}

The TCGA provisional cohort comprises 499 prostate cancer samples. In this study, we evaluated the genomic and transcriptomic profiles of 491 prostate cancer specimens. The TCGA cohort is composed by tumor samples obtained from different centers located in the United States (85.3\%), Germany (11\%), Australia (1.8\%), United Kingdom (1.4\%), and Brazil (0.4\%). We downloaded level 
3 RNA sequencing (RNAseq), array Comparative Genomic Hybridization (aCGH), and single nucleotide variation (SNV), and clinical data from the TCGA data portal (https://portal.gdc.cancer.gov/). Data normalization and segmentation were carried out in Nexus Copy Number 8.0 and Nexus Expression 3.0 (Biodiscovery, Santa Clara). SNV data was analyzed in R v3.4.2. Statistical analyses were carried out in R v3.4.2.

\section{Classification of PTEN deletions}

We first evaluated the presence or absence of PTEN deletions through analysis of aCGH data. In this analysis, samples were classified according to the presence of loss of one copy of PTEN gene (hemizygous) or loss of both copies of the PTEN gene (homozygous). Each deletion was considered separately in all tumors with homozygous deletions. We performed a supervised SCNA classification using Nexus Copy Number 8.0 to visualize and map the respective sizes of each PTEN deletion based on the distance between the positions of the copy number transitions along chromosome 10. In this analysis, we considered the largest deletion size when there was both a hemi- and a homozygous PTEN deletions with divergent lengths in the same tumor. A supervised SCNA classification was then performed using Nexus Copy Number 8.0 to visualize and map the respective sizes of each PTEN deletion based on the distance between the positions of the copy number transitions along chromosome 10. The five deletion subtypes were defined by the clustering of their respective size distributions along chromosome 10.

To investigate the presence of LCRs around the breakpoint regions, we searched the genomic position of the chromosome 10 deletion of each patient using the segmental duplication track of UCSC genome browser (http://genome.ucsc.edu browser; Human Genome Build 37). The analysis was carried out by using known LCRs (segmental duplication $>1 \mathrm{~kb}$ of non-repeat masked sequence with over $90 \%$ similarity) through Galaxy platform (https://usegalaxy.org/) [53, 54]. Further, the number of LCRs with high similarity $(>90 \%)$ and in the same orientation were counted for the upper and lower breakpoints of each sample.

\section{Genomic and chromosomal instability analysis}

We evaluated the effect of the different PTEN deletions on chromosomal and genomic instability. Chromosomal instability parameters were obtained from Nexus Copy Number 8.0. We evaluated the percentage of genome altered (ratio of the total length of all gain and loss calls by the length of the genome) and the total number of SCNAs (number of gains and losses events) for each tumor sample. No loss of heterozygosity or allelic imbalances were considered for the calculation of the percentage of genome altered and the total number of SCNAs. The genomic instability parameters were obtained through analysis of single nucleotide variants (SNVs). We performed an analysis of the total number of mutations in the genome, which included frameshift deletions and insertions, in-frame deletions, missense mutations, and splice-site. We also performed the analysis of the most significantly mutated genes through the MutSigCV algorithm [55]. Tumor heterogeneity levels were accessed through the mutant-allele tumor heterogeneity (MATH), which is the ratio of the width to the center of distribution of mutant-allele fractions among tumor-specific mutated loci [56].

\section{SCNA and transcriptome analysis}

Significant genomic changes were assessed by comparing the SCNA landscape of each group of PTEN deletion type through Nexus Copy Number 8.0. Differential SCNA calls between the compared groups were observed through the application of Fisher Exact Test with $P$-value $=0.05$ and alteration threshold percentage equal to $25 \%$. To access the genes associated with cancer pathways that were in regions of loss or gain, we analyzed the Cancer Gene Census feature from Nexus Copy Number 8.0. This feature generates a list of cancerrelated genes for each SCNA call.

For identification of differentially expressed genes between different PTEN deletion subtypes, matched RNAseq and aCGH data were analyzed. From 20,532 RNAseq probes, low variance probes $(<0.2)$ were filtered, resulting in 6081 probes. We then evaluated the expression of the 6081 genes and compared their expression profiles between each group of PTEN deletion subtypes with PTEN intact samples. Differentially expressed genes were obtained through Fisher Exact test through a logratio threshold of 0.1 and multiple test correction (FDR - Benjamini Hochberg, Q $<0.01$ ).

Further, we conducted an enrichment analysis of all differentially expressed genes obtained by comparing each deletion type with PTEN intact tumors. Pathway analysis was conducted through Database for Annotation, Visualization, and Integrated Discovery (DAVID, http://www.david.niaid.nih.gov) (version 6.8). The gene list for each deletion was imputed in DAVID, and Functional Annotation Charts were downloaded and analyzed through Cytoscape $3.0 \quad$ (http://www.cytoscape.org). Enrichment node construction was performed through Enrichment Map plugin (http://apps.cytoscape.org/apps/ enrichmentmap) for Cytoscape 3.0 using default options.

\section{Effect of the deletion subtypes in clinical parameters}

Analysis of the effect of the different PTEN deletion subtypes on clinical parameters was carried out in $R$ 
v3.4.2. We performed Chi-square tests for categorical data and Kruskal-Wallis tests for continuous clinical data. When significant associations were found by Chi-square analysis, we conducted univariate logistic regression analysis for the particular variable. We investigated the effect of each deletion type in the prediction of extraprostatic extension, seminal vesicle invasion, disease recurrence (defined the presence of at least one of the following events after radical prostatectomy: distant metastasis, local metastasis, biochemical recurrence, or new primary tumor), Gleason score, pathological $\mathrm{T}$ and $\mathrm{N}$, age at diagnosis, time to disease recurrence, and race. Additionally, log-rank test and Kaplan Meier curves were applied with disease recurrence as the endpoint. We also conducted univariate and multivariate Cox Regression models (Survival package) for the evaluated parameters. The comparisons were considered significantly different when $P$-value was $\leq 0.05$.

\section{Additional files}

Additional file 1: Effect of the copy number variation of PTEN gene in the chromosomal and genomic instability parameters. The boxplots show A - the total number of mutations, B - percentage of genome altered, C MATH score, and D - total number of SCNAs. PTEN homozygous deletions show an apparent effect on the SCNA and mutational landscapes, observed by an increased percentage of genome altered, total number of SCNAs, and total number of mutations. *Kruskal-Wallis test, $P$-value $<0.05$. (PNG $91 \mathrm{~kb}$ )

Additional file 2: Incidence of hemi- and homozygous deletions of PTEN per deletion subtype. (DOCX $12 \mathrm{~kb}$ )

Additional file 3: List of the genes in the deleted regions from chr10 for each deletion subtype. (DOCX $18 \mathrm{~kb}$ )

Additional file 4: Effect of PTEN inactivation in the aneuploidy and mutational landscapes in prostate cancer. Tumors with one allele inactive exhibit either a point mutation or one allele deletion (hemizygous deletion) of PTEN gene, resulting in reduced protein. Tumors with both alleles inactive exhibit either both copies of PTEN deleted (homozygous deletion) or one allele loss (hemizygous deletion) plus a point mutation in the remaining allele, resulting in an expected total loss of protein. From the 491 tumors, 367 were PTEN intact, 6 exhibited both copies of PTEN plus a point mutation, 62 presented hemizygous deletion of PTEN, 12 presented hemizygous deletion and one point mutation in the remaining PTEN allele, and 44 presented homozygous deletions of PTEN. SCNA - somatic copy number alteration. (PNG $84 \mathrm{~kb}$ )

Additional file 5: Boxplot showing the differences in PTEN mRNA expression for PTEN deletions. Kruskal-Wallis test was applied to identify significant differences in PTEN mRNA expression between the groups. (A) PTEN homozygous deletions showed the lowest levels of PTEN mRNA expression. (B) All PTEN deletion subtypes presented a significant decline in PTEN mRNA expression when compared to PTEN intact tumors. We did not observe any significant differences in PTEN mRNA expression levels within the subtype group. SI - Small Interstitial, LI - Large Interstitial, LP Large Proximal, LT - Large Terminal, E - Extensive. (PNG 66 kb)

Additional file 6: Clinical and pathological characterization for each deletion type. (DOCX $16 \mathrm{~kb}$ )

Additional file 7: Kaplan Meier plots and log-rank analysis of disease recurrence for tumors with distinct PTEN deletions in prostate cancer. (A) Log-rank test showed a significant difference between tumors with PTEN deletions and PTEN intact. (B) We did not observe a significant difference between the deletion subtypes through log-rank analysis. (PNG 978 kb)

\section{Abbreviations}

FISH: Fluorescence in situ hybridization; FoSTeS: Fork stalling and template switching; LCR: Low copy repeat; NAHR: Non-allelic homologous recombination; NHEJ: Non-homologous end-joining (NHEJ);

PTEN: Phosphatase and tensin homolog; SCNA: Somatic copy number alterations

\section{Funding}

JAS was supported by São Paulo Research Funding Agency (FAPESP) grant no. 2015/09111-5 and CNPq Bolsa Produtividade em Pesquisa - Nível: PQ-1B grant no. 306864/2014-2. TV and DGT are funded by FAPESP grants no. 2015/22785-5 and 2016/02730-4, respectively.

\section{Availability of data and materials}

The datasets analyzed during the current study are available in the TCGA repository [https://portal.gdc.cancer.gov].

\section{Authors' contributions}

TV conducted all bioinformatics and statistical analyses, interpreted all data, and wrote the manuscript; DGT performed MutSig and MATH score analyses; JAS supervised the study. All authors read and approved the final manuscript.

\section{Ethics approval and consent to participate}

Data obtained from the TCGA open-access database was collected from tumors of patients who provided informed consent based on the guidelines from the TCGA Ethics, Law and Policy Group.

\section{Consent for publication}

All patients included in the TCGA public domain database consented for publication as detailed in [https://cancergenome.nih.gov/abouttcga/policies/ informedconsent].

\section{Competing interests}

The authors declare that they have no competing interests.

\section{Publisher's Note}

Springer Nature remains neutral with regard to jurisdictional claims in published maps and institutional affiliations.

\section{Author details}

'Department of Genetics, Ribeirão Preto Medical School, University of São Paulo, Ribeirão Preto, Brazil. ${ }^{2}$ Deparment of Gynecology and Obstetrics, Clinical Hospital of Ribeirão Preto, Ribeirão Preto, Brazil. ${ }^{3}$ Department of Pathology and Legal Medicine, Ribeirão Preto Medical School, University of São Paulo, 3900 Bandeirantes Avenue, Monte Alegre, Ribeirão Preto, São Paulo 14040-900, Brazil. ${ }^{4}$ Department of Pathology and Molecular Medicine, Queen's University, Kingston, Canada.

Received: 10 November 2017 Accepted: 1 December 2017 Published online: 03 January 2018

\section{References}

1. Howlader N, Noone AM, Krapcho M, Miller D, Bishop K, Kosary CL et al. SEER Cancer Statistics Review, 1975-2014, National Cancer Institute 2017.

2. Verhagen P, van Duijn P, Hermans K, Looijenga L, van Gurp R, Stoop H, et al. The PTEN gene in locally progressive prostate cancer is preferentially inactivated by bi-allelic gene deletion. J Pathol. 2006;208:699-707.

3. Mithal P, Allott E, Gerber L, Reid J, Welbourn W, Tikishvili E, et al. PTEN loss in biopsy tissue predicts poor clinical outcomes in prostate cancer. Int J Urol. 2014;21:1209-14.

4. Troyer DA, Jamaspishvili T, Wei W, Feng Z, Good J, Hawley S, et al. A multicenter study shows PTEN deletion is strongly associated with seminal vesicle involvement and extracapsular extension in localized prostate cancer. Prostate. 2015;75:1206-15.

5. Picanço-Albuquerque CG, Morais CL, Carvalho FLF, Peskoe SB, Hicks IL, Ludkovski O, et al. In prostate cancer needle biopsies, detections of PTEN loss by fluorescence in situ hybridization (FISH) and by immunohistochemistry $(\mathrm{IHC})$ are concordant and show consistent association with upgrading. Virchows Arch. 2016;468:607-17. 
6. Fontugne J, Lee D, Cantaloni C, Barbieri CE, Caffo O, Hanspeter E, et al Recurrent prostate cancer genomic alterations predict response to brachytherapy treatment. Cancer Epidemiol Biomark Prev. 2014;23:594-600.

7. Silva MP, Barros-Silva JD, Ersvær E, Kildal W, Hveem TS, Pradhan M, et al. Cancer prognosis defined by the combined analysis of $8 q$, PTEN and ERG. Transl Oncol. 2016:9:575-82.

8. Hou S-Q, Ouyang M, Brandmaier A, Hao H, Shen WH. PTEN In the maintenance of genome integrity: from DNA replication to chromosome segregation. BioEssays. 2017;39:1700082

9. Williams $J$, Greer PA, Squire JA. Recurrent copy number alterations in prostate cancer: an in silico meta-analysis of publicly available genomic data. Cancer Genet Elsevier Inc. 2014;207:474-88.

10. Hanahan D, Weinberg RA. Hallmarks of cancer: the next generation. Cell. 2011;144:646-74.

11. Tapia-Laliena MA, Korzeniewski N, Hohenfellner M, Duensing S. High-risk prostate cancer: a disease of genomic instability. Urol Oncol Semin Orig Investig. 2014;32:1101-7.

12. Yoshimoto M, Ludkovski O, Degrace D, Williams JL, Evans A, Sircar K, et al. PTEN genomic deletions that characterize aggressive prostate cancer originate close to segmental duplications. Genes Chromosom Cancer. 2012; 51:149-60.

13. Biscotti MA, Olmo E, Heslop-Harrison JSP, Repetitive DNA. In eukaryotic genomes. Chromosom Res. 2015;23:415-20.

14. Deloukas P, Matthews LH, Ashurst J, Burton J, Gilbert JGR, Jones M, et al. The DNA sequence and comparative analysis of human chromosome 20. Nature. 2001;414:865-71.

15. Stankiewicz P, Kulkarni S, Dharmadhikari AV, Sampath S, Bhatt SS, Shaikh TH, et al. Recurrent deletions and reciprocal duplications of 10q11.21q11.23 including CHAT and SLC18A3 are likely mediated by complex low-copy repeats. Hum Mutat. 2012;33:165-79.

16. Kloosterman WP, Guryev V, van Roosmalen M, Duran KJ, de Bruijn E, Bakker SCM, et al. Chromothripsis as a mechanism driving complex de novo structural rearrangements in the germline. Hum Mol Genet. 2011;20:1916-24.

17. Kass EM, Moynahan ME, Jasin M. When genome maintenance goes badly awry. Mol Cell. 2016;62:777-87.

18. Bishop AJ, Schiestl RH, et al. Hum Mol Genet. 2000:9:2427-334.

19. Hastings PJ, Lupski JR, Rosenberg SM, Ira G. Mechanisms of change in gene copy number. Nat Rev Genet. 2009;10:551-64.

20. Yoshimoto M, Cunha IW, Coudry RA, Fonseca FP, Torres CH, Soares FA, et al. FISH analysis of 107 prostate cancers shows that PTEN genomic deletion is associated with poor clinical outcome. Br J Cancer. 2007;97:678-85.

21. Choucair K, Ejdelman J, Brimo F, Aprikian A, Chevalier S, Lapointe JPTEN. Genomic deletion predicts prostate cancer recurrence and is associated with low AR expression and transcriptional activity. BMC Cancer. 2012;12: 543-51.

22. Murphy SJ, Karnes RJ, Kosari F, Castellar BERP, Kipp BR, Johnson SH, et al. Integrated analysis of the genomic instability of PTEN in clinically insignificant and significant prostate cancer. Mod Pathol. 2016;29:143-56.

23. Fraser $M$, Sabelnykova $W$, Yamaguchi TN, Heisler LE, Livingstone J, Huang V, et al. Genomic hallmarks of localized, non-indolent prostate cancer. Nature. 2017;541:359-64.

24. Abeshouse A, Ahn J, Akbani R, Ally A, Amin S, Andry CD, et al. The molecular taxonomy of primary prostate cancer. Cell. 2015;163:1011-25.

25. Lotan TL, Wei W, Ludkovski O, Morais CL, Guedes LB, Jamaspishvili T, et al. Analytic validation of a clinical-grade PTEN immunohistochemistry assay in prostate cancer by comparison with PTEN FISH. Mod Pathol. 2016;29:904-14.

26. Cuzick J, Yang ZH, Fisher G, Tikishvili E, Stone S, Lanchbury JS, et al. Prognostic value of PTEN loss in men with conservatively managed localised prostate cancer. Br J Cancer. 2013;108:2582-9.

27. Chaisson MJP, Wilson RK, Eichler EE. Genetic variation and the de novo assembly of human genomes. Nat Rev Genet. 2015;16:627-40.

28. de Smith AJ, Walters RG, Coin LJM, Steinfeld I, Yakhini Z, Sladek R, et al. Small deletion variants have stable breakpoints commonly associated with alu elements. PLoS One. 2008:3:1-11.

29. Duijf PHG, Benezra R. The cancer biology of whole-chromosome instability. Oncogene. 2013;32:4727-36.

30. Shen WH, Balajee AS, Wang J, Wu H, Eng C, Pandolfi PP, et al. Essential role for nuclear PTEN in maintaining chromosomal integrity. Cell. 2007;128:157-70.

31. Bassi C, Ho J, Srikumar T, Dowling RJO, Gorrini C, Miller SJ, et al. Nuclear PTEN controls DNA repair and sensitivity to genotoxic stress. Science. 2013: 341:395-9.
32. Kurfurstova D, Bartkova J, Vrtel R, Mickova A, Burdova A, Majera D, et al. DNA damage signalling barrier, oxidative stress and treatment-relevant DNA repair factor alterations during progression of human prostate cancer. Mol Oncol. 2016:10:879-94.

33. Davoli T, AW X, Mengwasser KE, Sack LM, Yoon JC, Park PJ, et al. Cumulative haploinsufficiency and triplosensitivity drive aneuploidy patterns and shape the cancer genome. Cell. 2013;155:948-62.

34. Solimini NL, Xu Q, Mermel CH, Liang AC, Schlabach MR, Luo J, et al. Recurrent hemizygous deletions in cancers may optimize proliferative potential. Science. 2012;337:104-9.

35. Sun Z, Huang C, He J, Lamb KL, Kang X, Gu T, et al. PTEN C-terminal deletion causes genomic instability and tumor development. Cell Rep. 2014:6:844-54.

36. Hopkins BD, Hodakoski C, Barrows D, Mense SM, Parsons REPTEN. Function: the long and the short of it. Trends Biochem Sci. 2015:39:183-90.

37. Zhao D, Lu X, Wang G, Lan Z, Liao W, Li J, et al. Synthetic essentiality of chromatin remodelling factor CHD1 in PTEN-deficient cancer. Nature. 2017; 542:484-8.

38. Isaacs WB, Carter BS, Debruyne FMJ, Schalken JA. Expression of the cellular adhesion molecule E-Cadherin is reduced or absent in high-grade prostate cancer. Cancer Res. 1992;52:5104-9.

39. Ni J, Cozzi P, Hao J, Beretov J, Chang L, Duan W, et al. Epithelial cell adhesion molecule (EpCAM) is associated with prostate cancer metastasis and chemo/radioresistance via the PI3K/Akt/mTOR signaling pathway. Int J Biochem Cell Biol. 2013;45:2736-48.

40. Seguin L, Desgrosellier JS, Weis SM, Cheresh DA. Integrins and cancer: regulators of cancer stemness, metastasis, and drug resistance. Trends Cell Biol. 2015;25:234-40.

41. Zhang Q, Liu S, Zhang Q, Xiong Z, Wang AR, Myers L, et al. Interleukin-17 promotes development of castration-resistant prostate cancer potentially through creating an immunotolerant and pro-angiogenic tumor microenvironment. Prostate. 2014;74:869-79.

42. Martin PL, Yin J-J, Seng V, Casey O, Corey E, Morrissey C, et al. Androgen deprivation leads to increased carbohydrate metabolism and hexokinase 2-mediated survival in Pten/Tp53-deficient prostate cancer. Oncogene. 2017 36:525-33.

43. Robles Al, Harris CC. Clinical outcomes and correlates of TP53 mutations and cancer. Cold Spring Harb Perspect Biol. 2010;2:1-15.

44. Bunz F, Kinzler KW, Vogelstein B, Lengauer C, Vogelstein B, Fauth C, et al. Targeted inactivation of p53 in human cells does not result in aneuploidy. Cancer Res. 2002;62:1129-33.

45. Macheret M, Halazonetis TD. DNA replication stress as a Hallmark of cancer. Annu Rev Pathol Mech Dis. 2015;10:425-48.

46. Nikolaev SI, Sotiriou SK, Pateras IS, Santoni F, Sougioultzis S, Edgren H, et al. A single-nucleotide substitution mutator phenotype revealed by exome sequencing of human colon adenomas. Cancer Res. 2012;72: 6279-89.

47. Ngeow J, Mester J, Rybicki LA, Ni Y, Milas M, Eng C. Incidence and clinical characteristics of thyroid cancer in prospective series of individuals with cowden and cowden-like syndrome characterized by germline PTEN, SDH, or KLLN alterations. J Clin Endocrinol Metab. 2011:96:2063-71.

48. Nizialek EA, Peterson C, Mester JL, Downes-Kelly E, Eng C. Germline and somatic KLLN alterations in breast cancer dysregulate G2 arrest. Hum Mol Genet. 2013;22:2451-61.

49. Kuehn HS, Caminha I, Niemela JE, Rao VK, Davis J, Fleisher TA, et al. FAS Haploinsufficiency is a common disease mechanism in the human autoimmune Lymphoproliferative syndrome. J Immunol. 2011;186:6035-43.

50. Khani F, Mosquera JM, Park K, Blattner M, O'Reilly C, MacDonald TY, et al. Evidence for molecular differences in prostate cancer between african American and Caucasian men. Clin Cancer Res. 2014:20:4925-34.

51. Lindquist KJ, Paris PL, Hoffmann TJ, Cardin NJ, Kazma R, Mefford JA, et al. Mutational landscape of aggressive prostate tumors in African American men. Cancer Res. 2016;76:1860-8.

52. Tosoian JJ, Almutairi F, Morais CL, Glavaris S, Hicks J, Sundi D, et al. Prevalence and prognostic significance of PTEN loss in African-American and European-American men undergoing radical prostatectomy. Eur Urol. European association of. Urology. 2017;71:697-700.

53. Shinawi M, Liu P, Kang SHL, Shen J, Belmont JW, Scott D, et al. Recurrent reciprocal $16 p 11.2$ rearrangements associated with global developmental delay, behavioural problems, dysmorphism, epilepsy, and abnormal head size. J Med Genet. 2010;47:332-41. 
54. Leite AJC, Pinto IP, Da Cruz E, Cunha DM, Ribeiro CL, Da Silva CC, et al. The identification of microdeletion and reciprocal microduplication in 22911.2 using high-resolution CMA technology. Biomed Res Int. 2016;2016:1-9.

55. Lawrence MS, Stojanov P, Polak P, Kryukov GV, Cibulskis K, Sivachenko A, et al. Mutational heterogeneity in cancer and the search for new cancerassociated genes. Nature. 2013;499:214-8.

56. Mroz EA, Rocco JW. MATH, A novel measure of intratumor genetic heterogeneity, is high in poor-outcome classes of head and neck squamous cell carcinoma. Oral Oncol. 2013;49:211-5.

Submit your next manuscript to BioMed Central and we will help you at every step:

- We accept pre-submission inquiries

- Our selector tool helps you to find the most relevant journal

- We provide round the clock customer support

- Convenient online submission

- Thorough peer review

- Inclusion in PubMed and all major indexing services

- Maximum visibility for your research

Submit your manuscript at www.biomedcentral.com/submit 